\title{
Repurposing propranolol as an antitumor agent in von Hippel-Lindau disease
}

\author{
Matthew J. Shepard, MD, ${ }^{1,2}$ Alejandro Bugarini, BS, ${ }^{1}$ Nancy A. Edwards, BA, ${ }^{1}$ Jie Lu, PhD, ${ }^{1,4}$ \\ Qi Zhang, MD, ${ }^{1,3}$ Tianxia Wu, PhD, ${ }^{1}$ Zhengping Zhuang, MD, PhD, ${ }^{1,3}$ and Prashant Chittiboina, MD ${ }^{1,4}$ \\ 'Surgical Neurology Branch, National Institute of Neurological Disorders and Stroke, National Institutes of Health, Bethesda, \\ Maryland; ' Department of Neurological Surgery, University of Virginia Health System, Charlottesville, Virginia; ${ }^{3}$ Neuro-Oncology \\ Branch, National Cancer Institute, National Institutes of Health, Bethesda; and ${ }^{4}$ Neurosurgery Unit for Pituitary and Inheritable \\ Diseases, National Institute of Neurological Disorders and Stroke, National Institutes of Health, Bethesda, Maryland
}

\begin{abstract}
OBJECTIVE Von Hippel-Lindau disease (VHL) is a tumor predisposition syndrome characterized by CNS hemangioblastomas (HBs) and clear cell renal cell carcinomas (RCCs) due to hypoxia-inducible factor activation (pseudohypoxia). Because of the lack of effective medical therapies for VHL, HBs and RCCs account for significant morbidity and mortality, ultimately necessitating numerous neurological and renal surgeries. Propranolol is an FDA-approved pan-beta adrenergic antagonist with antitumor effects against infantile hemangiomas $(\mathrm{IHs})$ and possibly VHL HBs. Here, the authors investigated the antitumor efficacy of propranolol against pseudohypoxia-driven VHL-HBs and VHL-RCCs.
\end{abstract}

METHODS Patient-derived VHL-associated HBs (VHL-HBs) or 786-0-VHL ${ }^{-/}$RCC cells were treated with clinically relevant concentrations of propranolol in vitro and assessed with viability assays, flow cytometry, quantitative real-time polymerase chain reaction, and western blotting. In vivo confirmation of propranolol antitumor activity was confirmed in athymic nude mice bearing 786-O xenograft tumors. Lastly, patients enrolled in a VHL natural history study (NCT00005902) were analyzed for incidental propranolol intake. Propranolol activity against VHL-HBs was assessed retrospectively with volumetric HB growth kinetic analysis.

RESULTS Propranolol decreased HB and RCC viability in vitro with $\mathrm{IC}_{50}$ (half maximal inhibitory concentration) values of $50 \mu \mathrm{M}$ and $200 \mu \mathrm{M}$, respectively. Similar to prior reports in infantile hemangiomas, propranolol induced apoptosis and paradoxically increased VEGF-A mRNA expression in patient-derived VHL-HBs and 786-O cells. While intracellular VEGF protein levels were not affected by propranolol treatment, propranolol decreased HIF expression in 786-0 cells (7.6-fold reduction, $p<0.005)$. Propranolol attenuated tumor progression compared with control $(33 \%$ volume reduction at 7 days, $p<0.005$ ) in 786-O xenografted tumor-bearing mice. Three patients (harboring 25 growing CNS HBs) started propranolol therapy during the longitudinal VHL-HB study. HBs in these patients tended to grow slower (median growth rate $27.1 \mathrm{~mm}^{3} /$ year vs $13.3 \mathrm{~mm}^{3} /$ year) during propranolol treatment $(p<0.0004)$.

CONCLUSIONS Propranolol decreases VHL-HB and VHL-related RCC viability in vitro likely by modulation of VEGF expression and by inducing apoptosis. Propranolol abrogates 786-O xenograft tumor progression in vivo, and retrospective clinical data suggest that propranolol curtails HB growth. These results suggest that propranolol may play a role in the treatment of VHL-related tumors.

https://thejns.org/doi/abs/10.3171/2018.5.JNS172879

KEYWORDS von Hippel-Lindau disease; VHL; hemangioblastoma; renal cell carcinoma; propranolol; translational medicine; oncology

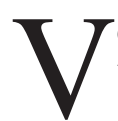
ON Hippel-Lindau (VHL) disease is an inherited tumor predisposition syndrome that is caused by germline mutations in the $V H L$ tumor suppressor gene found on the short arm of chromosome $3 .^{5,11,17} \mathrm{~Pa}$ tients with VHL have a propensity to develop hemangio- blastomas (HBs) of the CNS and retina as well as renal cell carcinomas (RCCs), endolymphatic sac tumors, pheochromocytomas, and pancreatic neuroendocrine tumors. ${ }^{5,17}$ Although the manifestations of the disorder can vary significantly across individuals, the pathophysiology of tumor

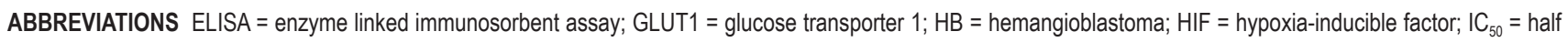
maximal inhibitory concentration; $I H=$ infantile hemangioma; $P B S=$ phosphate-buffered saline; $R C C=$ renal cell carcinoma; $R T-P C R=$ real-time polymerase chain reaction; VEGF = vascular endothelial growth factor; VHL = von Hippel-Lindau; VHL-HB = VHL-related HB; VHL-RCC $=$ VHL-related RCC.

SUBMITTED November 15, 2017. ACCEPTED May 10, 2018.

INCLUDE WHEN CITING Published online October 26, 2018; DOI: 10.3171/2018.5.JNS172879. 
formation for RCCs, HBs, and pancreatic neuroendocrine tumors is believed to result from inappropriate upregulation of hypoxia response elements. ${ }^{8,25}$ Patients with VHL harbor a quantitative or qualitative deficiency of the VHL protein, which, under normoxic conditions, fosters the degradation of hypoxia-inducible factors (HIFs), ${ }^{8,17,36}$ In susceptible tissues, loss of heterozygosity of the wild-type $V H L$ allele leads to constitutive, inappropriate upregulation of HIFs that upregulate expression of a variety of genes, including erythropoietin, vascular endothelial growth factor (VEGF), and transforming growth factor-alpha, which are believed to play important roles in tumorgenesis. $3,5,9,11,17$

As a result of the development of a variety of benign and malignant neoplasms, patients with VHL have a median survival ranging between 40 and 52 years, the shortest of any tumor predisposition syndrome. ${ }^{34}$ The leading causes of morbidity and mortality in patients with VHL are RCC and CNS HB. ${ }^{5,8,16}$ Both RCC and HB are common manifestations of VHL, occurring in $30 \%$ and $72 \%$ of affected individuals, respectively. ${ }^{8,17}$ The treatment for VHL-related RCC (VHL-RCC) and HBs is surgery. As patients with VHL can develop upward of 600 RCC foci per kidney and 10 or more symptomatic HBs over the course of their lifetimes, they often require multiple renal and neurological surgeries. ${ }^{8,16,33}$ Although there have been reports on the efficacy of certain antitumor agents for VHL-related HBs (VHL-HBs), no systemic therapy has gained widespread use in curtailing the progression of VHL-HBs or VHL-RCCs..$^{22,28,29}$ Thus, the identification of a well-tolerated, safe, and easily administered systemic therapy that curbs VHL-HB or VHL-RCC progression would represent a significant advancement in the treatment of patients with VHL disease.

Léauté-Labrèze et al. demonstrated that propranolol, an FDA-approved, nonselective, $\beta 1$ and $\beta 2$ adrenergic antagonist used for the treatment of hypertension and cardiac arrhythmias, is effective in treating infantile hemangiomas (IHs), i.e., benign, vascular tumors of infancy. ${ }^{12,13}$ Given the histological and pathophysiological similarities between IHs and VHL-HBs, it has been hypothesized that propranolol may be an effective treatment in patients with VHL disease. Albiñana et al. showed that propranolol is able to reduce HIF- $1 \alpha$ expression in patient-derived HB cells while inducing apoptosis and decreasing cell viability in vitro. ${ }^{2}$ As a result, we sought to confirm these findings and further expand on these results to determine if propranolol may harbor broader treatment effects in VHL-HBs and VHL-RCCs.

\section{Methods}

\section{Clinical Sample Collection}

HB samples were obtained under a National Institutes of Health institutional review board-approved protocol (clinicaltrials.gov identifier NCT00060541). All specimens were obtained from individuals undergoing surgery of symptom-producing HBs. Informed consent was obtained from all patients.

\section{HB Stromal Cell Extraction and Cell Culture}

$\mathrm{HB}$ tumor cells were isolated as previously described..$^{24,30}$
Briefly, isolated $\mathrm{HB}$ cells were incubated at $37^{\circ} \mathrm{C}$ for 24 hours after isolation in DMEM supplemented with $10 \%$ fetal bovine serum, L-glutamine, penicillin, streptomycin, and F-12K. All subsequent experiments were performed within 24-48 hours of HB extraction. The 786-O RCC cell line was obtained from America Type Culture Collection and grown per the distributer's specification.

\section{Cell Viability Assays}

Cells $\left(1 \times 10^{4}\right)$ were plated on flat-bottom 96-well plates and subsequently exposed to $0-400 \mu \mathrm{M}(S)-(-)$-propranolol hydrochloride (Sigma-Aldrich) for 24-48 hours at $37^{\circ} \mathrm{C},{ }^{7,38}$ After incubation, the Cell Counting Kit-8 cytotoxicity assay (Dojindo Molecular Technologies) was subsequently performed according to the manufacturer's protocol. An ELx800 96-well spectrometer (BioTek Instruments) was used to perform all absorbance measurements.

\section{RNA Extraction and Quantitative Real-Time Polymerase Chain Reaction}

RNA was extracted from cells using the RNeasy Mini Kit (Qiagen) according to manufacturer's instructions. Complementary DNA libraries were subsequently created using SuperScript III First-Strand Synthesis SuperMix (Invitrogen Life Technologies). Real-time polymerase chain reaction (RT-PCR) was then performed using QuantStudio 6 Flex RT-PCR system (Applied Biosystems). Gene expression was determined using primers designed for vascular endothelial growth factor (VEGF, Qiagen), glucose transporter 1 (GLUT1, Qiagen), and hypoxia-inducible factor $1 \alpha$ (HIF1 $\alpha$, Qiagen), with $\beta$-actin as internal control (Sigma-Aldrich). In all instances, the Power SYBR Green PCR Master Mix was utilized (Applied Biosystems).

\section{Apoptosis Assays}

The 786-O cells were cultured in the presence of $0-200$ $\mu \mathrm{M}(S)-(-)$-propranolol hydrochloride (Sigma-Aldrich) for $24-48$ hours at $37^{\circ} \mathrm{C}$. The Vybrant Apoptosis Assay Kit \#5 (Hoechst 33342/propidium iodide, ThermoFisher Scientific) was then used to determine the percentage of necrotic and apoptotic cells in culture according to the manufacturer's specification.

\section{Immunoblot Analysis}

For protein extraction, whole-cell lysates were collected in radioimmunoprecipitation assay buffer containing a protease inhibitor cocktail and centrifuged at $15,295 \mathrm{~g}$ for 15 minutes at $4^{\circ} \mathrm{C}$. Protein concentration was determined by Nanodrop 2000 (Thermo Fisher Scientific). Proteins were electrophoretically separated on 4\%-12\% Bis-Tris gels (Invitrogen) and subsequently electroblotted onto polyvinylidene difluoride membranes with the TransBlot Transfer Turbo System (Bio-Rad). After blocking, the membranes were probed with anti-VEGF (Thermo Fisher Scientific) or anti-HIF2 $\alpha$ (sc-46691, Santa Cruz), and the immunoreactive signal was detected with the ChemiDoc MP Imaging System (Bio-Rad). Densitometry was performed using Image Lab software (Bio-Rad). 


\section{6-0 Xenograft Mouse Model}

All animal experiments were approved by the National Institute of Neurological Disorders and Stroke Animal Care and Use Committee. Five- to 6-week-old $(\mathrm{n}=10)$ female athymic nude mice (Jackson Laboratory) were injected in the right flank with $5 \times 10^{6} 786-\mathrm{O}$ cells as previously described..$^{10}$ Tumor volume was determined using the formula $\mathrm{V}=(\mathrm{L} \times \mathrm{W}) / 2$, where $\mathrm{L}$ and $\mathrm{W}$ represent the length and width of the tumor, respectively. ${ }^{10}$ When tumors grew to between 80 and $150 \mathrm{~mm}^{3}$, the mice were randomized to receive daily intraperitoneal injections of propranolol $(20 \mathrm{mg} / \mathrm{kg})$ or phosphate-buffered saline (PBS) ${ }^{31}$ Flank tumor growth was monitored over time. At the end of the study, blood was collected from mice, and the serum was analyzed for levels of mouse and human VEGF-A using VEGF ELISA kits (Thermo Fisher Scientific) per the manufacturer's instructions.

\section{Immunohistochemistry}

When PBS or propranolol-treated mice reached their endpoint (tumor volumes greater than $1000 \mathrm{~mm}^{3}$ or evidence of ulceration), the flank tumors were harvested and fixed in formalin. The fixed tumors were paraffin embedded, and blocks were sectioned at 5- $\mu$ m thickness. An automated immunostainer (Bond-Max, Leica) was used for reproducible staining of paraffin sections. Tissue was probed using antibodies against HIF2 $\alpha$ (Bioss), VEGF (Proteintech), and GLUT1 (Proteintech). Antibodies were optimized using positive control tissue with high $\mathrm{pH}$ (EDTA) epitope retrieval.

\section{Incidental use of Propranolol}

Patients with VHL disease and HBs who were enrolled in a natural history study (clinicaltrials.gov identifier NCT00005902) were retrospectively assessed for oral propranolol usage. Patients who underwent clinical and radiographic follow-up before and after propranolol implementation were assessed. HBs were assessed using postcontrast T1-weighted MRI. HB volumes were calculated using a modified ellipse formula. ${ }^{16,18}$ The growth rates of tumors with evidence of growth were determined before and after propranolol therapy was implemented as previously described. ${ }^{16}$

\section{Statistical Analysis}

All in vitro studies were subjected to at least 3 independent biological experiments. Data are presented as the mean \pm SEM. Statistical analysis was performed using Prism Software (GraphPad). One-way ANOVA was used for comparing results of RT-PCR. The t-test was used for continuous variables for in vitro and in vivo experiments; $\mathrm{p}<0.05$ was considered statistically significant.

For the HB-volumetric analysis, the volumetric growth rate per year during a period was estimated as the difference in tumor volume between the first and last clinic visit divided by the tumor volume at the first visit and the duration of tumor follow-up (in years). For each tumor, the growth rate per year was determined before and after propranolol implementation. Since each patient harbored multiple tumors and propranolol was a within-subject fac- tor, a nested model was applied to model both patient and tumor (nested within patient) as random effects while propranolol was treated as a fixed effect. ${ }^{15}$ Natural logarithm transformation was applied to the growth rates, and the Shapiro-Wilk test was used to test the normality of the residuals. SAS (version 9.4, SAS Institute) was used for the above analyses; $\mathrm{p}<0.05$ was considered statistically significant.

\section{Results}

\section{Propranolol Reduces the Viability of Patient-Derived VHL-HB Cells}

In order to confirm the results of Albiñana et al., patient-derived $\mathrm{HB}$ cells were cultured from tumors resected from 4 separate individuals with VHL disease. Patientderived HB primary cultures were then treated with propranolol $(0-400 \mu \mathrm{M})$ for 24 or 48 hours. Cultured HB viability tended to decrease in both a dose- and time-dependent manner (Fig. 1A-D). HB viability was reduced by $50 \%$ (half maximal inhibitory concentration; $\mathrm{IC}_{50}$ ) with exposure to $50 \mu \mathrm{M}$ of propranolol within 24 hours. Cells cultured with at least $50 \mu \mathrm{M}$ of propranolol also demonstrated morphological changes within 48 hours after exposure (Fig. 1B). Cells became rounded, which has been observed in cells undergoing apoptosis in the presence propranolol. ${ }^{2}$ Treatment with $100 \mu \mathrm{M}$ of propranolol led to the loss of viability of almost all cells in culture at 24 and 48 hours (Fig. 1C and D).

\section{Propranolol Induces HIF, VEGF, and GLUT-1 mRNA Expression in VHL-HB Cells}

Propranolol leads to a discordant increase in VEGF mRNA expression while also reducing intracellular VEGF protein expression due to interference with VEGF mRNA translation. ${ }^{7}$ However, other studies have demonstrated that propranolol may lead to a decrease in VEGF mRNA and protein expression. ${ }^{2}$ We therefore investigated the effect of propranolol on gene expression within the hypoxia pathway in 2 patient-derived HB primary cultures. On treatment with propranolol $(50 \mu \mathrm{M}$ and $100 \mu \mathrm{M})$ for 24 or 48 hours, HIF1 $\alpha$, VEGF-A, and GLUT1 mRNA were overexpressed (Fig. 1E and F). The largest increase in expression was evident after 48 hours of treatment with $100 \mu \mathrm{M}$ of propranolol (approximately 3.5 -fold increase in HIF1 $\alpha$, approximately 15-fold increase in VEGF expression, and approximately 15-fold increase in GLUT1 expression; $\mathrm{p}<0.0001$ ).

\section{Propranolol Reduces the Viability of a VHL-/- RCC Cell Line by Inducing Apoptosis}

As VHL-HBs are not malignant tumors and no established cell line for HBs exist, we further explored the mechanisms of propranolol activity in the immortalized human RCC cell line (786-O), which lacks a functional $V H L$ gene $\left(V_{H L}^{--}\right)$. Thus, the 786-O line represents a useful probe for studying VHL-related tumors in vitro. ${ }^{36}$ Propranolol decreased 786-O viability in vitro, albeit with an $\mathrm{IC}_{50}$ at $200 \mu \mathrm{M}$ within 24 hours (Fig. 2A). As propranolol has been shown to induce apoptosis in VHL-HBs and IHs, we investigated whether the decreased viability observed 

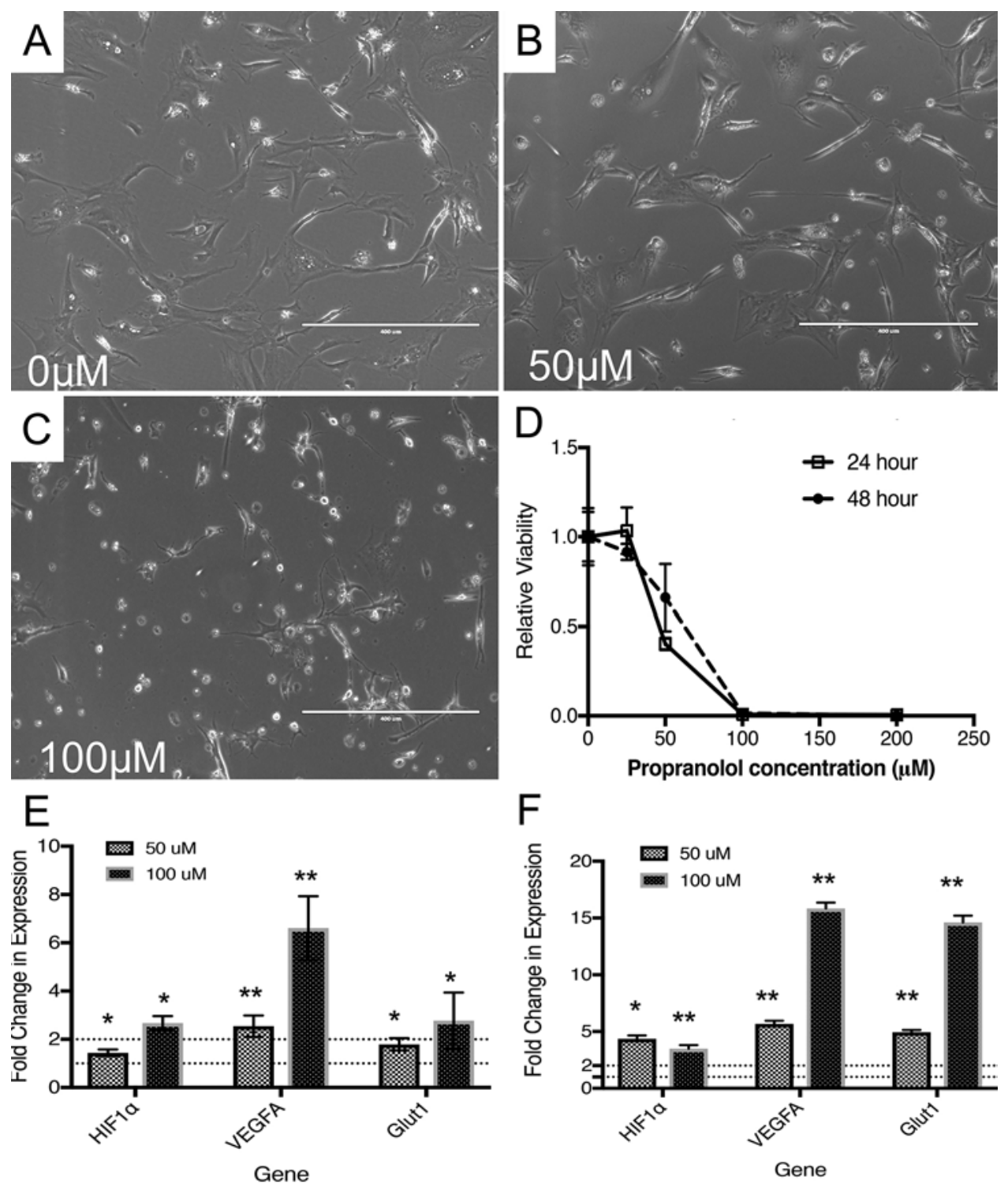

FIG. 1. Propranolol leads to decreased HB viability ex vivo. Patient-derived HB cells were cultured and treated with propranolol $(0-400 \mu \mathrm{M})$. A-C: Representative photomicrographs $(\times 10)$ of treated HB cells exposed to $0 \mu \mathrm{M}(\mathrm{A}), 50 \mu \mathrm{M}(\mathrm{B})$, and $100 \mu \mathrm{M}(\mathrm{C})$ of propranolol are shown. Cells exposed to propranolol become rounded $(B)$ prior to completely detaching from the cultureware $(C)$ with increasing concentrations of propranolol. D: Representative viability assays are shown. Propranolol decreases HB viability with an approximate $\mathrm{IC}_{50}$ of $50 \mu \mathrm{M}$ after 24 or 48 hours of propranolol exposure $(\mathrm{n}=3$ for each data point, experiments repeated over 4 different $\mathrm{HB}$ specimens). $\mathrm{E}$ and $\mathrm{F}$ : Gene expression analysis of the pseudohypoxia-signaling pathway was performed using RT-PCR ( $E$ and F). Propranolol induces HIF-1 $\alpha$, VEGF-A, and GLUT1 mRNA levels at 24 (E) and 48 (F) hours, which has been observed previously in propranolol-treated IHs $\left(n=3\right.$, for each data point). ${ }^{*} p<0.05,{ }^{* *} p<0.0001$.

in propranolol-treated 786-O cells could be secondary to apoptosis. Using the Hoechst propidium iodide assay, we found that there was a 4.5 -fold increase in the population of cells undergoing apoptosis when exposed to $200 \mu \mathrm{M}$ of propranolol for 24 hours (Fig. $2 \mathrm{~B}, \mathrm{p}<0.0001$ ). Consistent with our viability assays, flow cytometry depicted a substantial reduction in the number of viable cells $(72 \%$ reduction, $\mathrm{p}<0.0001)$. While this was partly explained by the increased rates of apoptosis, 786-O cells exposed to propranolol had a 6-fold increase in the percentage of necrotic cells $(\mathrm{p}<0.0001)$, suggesting a potential direct toxic effect of propranolol on these cells.

\section{Propranolol Decreases HIF Expression Without Affecting VEGF Expression in 786-0 Cells}

As propranolol has been shown to affect the hypoxiasignaling pathway in IHs, we investigated whether HIF, VEGF, or GLUT1 expression was affected in 786-O cells treated with propranolol. As was the trend in VHL-HBs, propranolol led to 1.9- and 1.4-fold increases in GLUT1 and HIF mRNA, respectively ( $p<0.05$, Fig. 2 C). In a less dramatic fashion compared with VHL-HBs, propranolol increased VEGF-A mRNA expression approximately 2-fold in 786-O cells (Fig. 2C). We tested whether 
A

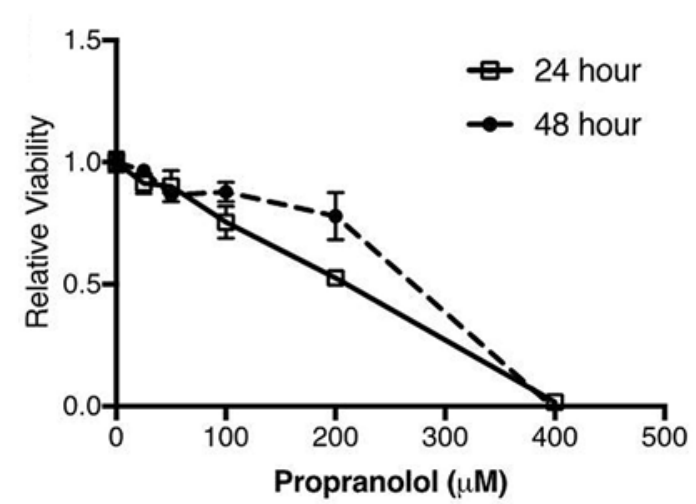

C

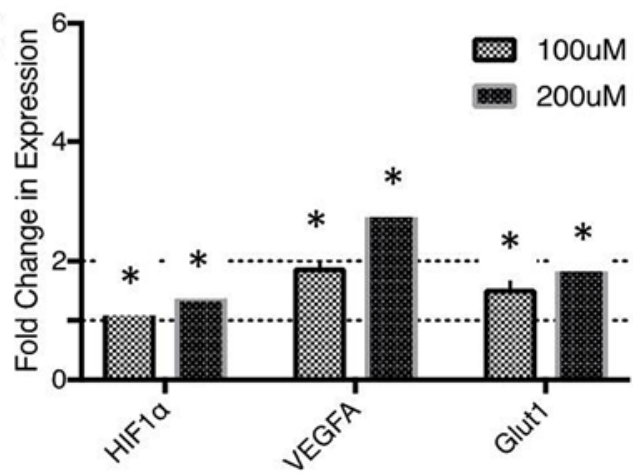

B

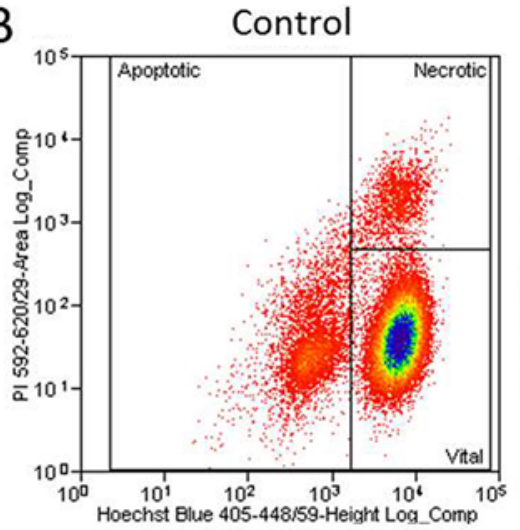

Control

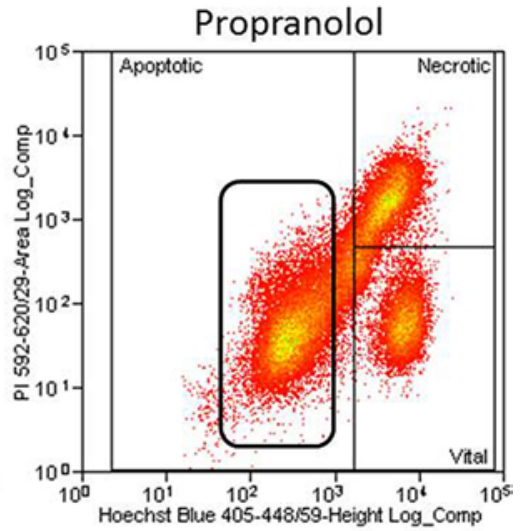

D

Control

$200 \mu \mathrm{M}$ Propranolol

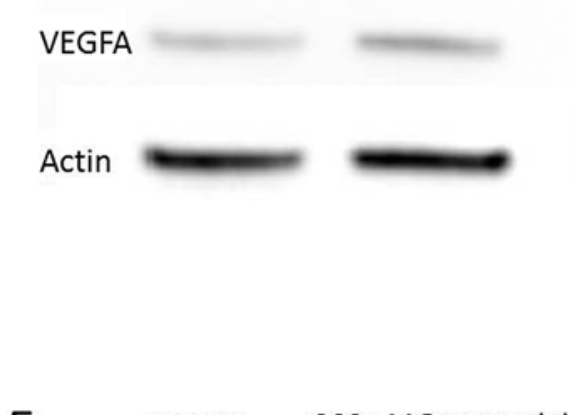

E Control $\quad 200 \mu \mathrm{M}$ Propranolol

HIF2 $\alpha$

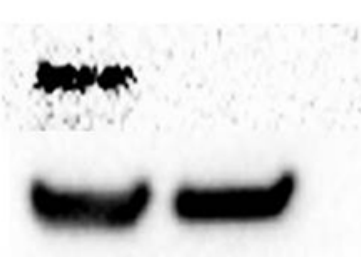

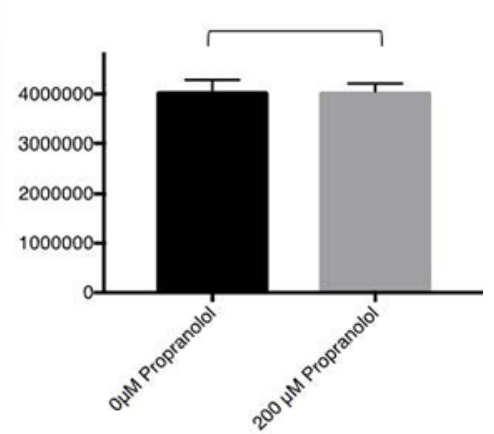

$p=0.99$

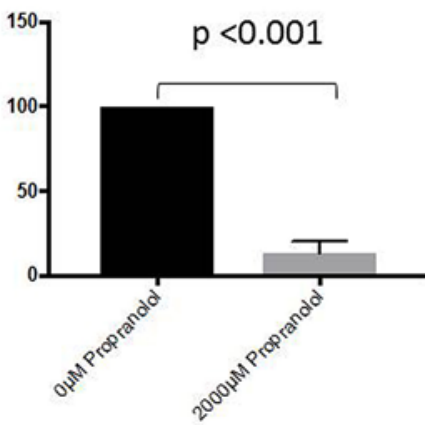

FIG. 2. Propranolol decreases RCC cell line viability in vitro by inducing apoptosis. A: Results of viability assays exhibit decreased 786-O viability at 24 or 48 hours in the presence of $200 \mu \mathrm{M}$ of propranolol. Biological triplicates were performed for each data point shown. B: Flow cytometry was used to investigate the degree of apoptotic, necrotic, and viable cells after treatment with 200 $\mu \mathrm{M}$ of propranolol. Results suggest that treated cells had a 4.5 -fold increase in rates of apoptosis $(p<0.0001)$, a 6 -fold increase in rates of necrosis $(p<0.0001)$, and a $72 \%$ reduction in viability $(n=3, p<0.0001)$. C: RT-PCR $(n=3)$ was then performed to investigate gene expression in 786-O cells exposed to $200 \mu \mathrm{M}$ of propranolol. There were increases in HIF-1 $\alpha$, VEGF-A, and GLUT1 mRNA levels in a dose-dependent manner. This is similar to results observed in IHs. Propranolol had no effect on intracellular VEGF-A protein levels, but did decrease HIF2 $\alpha$ expression in 786-O cells $(n=3)$. D and E: Representative western blots for VEGF (D) and HIF2 $\alpha(E)$ are shown. Normalized relative intensity values are shown next to representative immunoblots. ${ }^{*} p<0.05$. Figure is available in color online only.

propranolol inhibits the translation of VEGF-A from its mRNA template. Surprisingly, unlike reports in VHLHBs and IHs, VEGF protein levels were unchanged (Fig. 2D), suggesting that at least in this RCC cell line, the changes in viability observed in 786-O cells seen with increasing propranolol concentrations are not affected by VEGF protein expression in vitro. As other authors have suggested that propranolol may decrease HIF expression in IHs, we assayed HIF2 $\alpha$ levels in 786-O cells exposed to propranolol, as HIF $2 \alpha$ is the predominant isoform found in 786-O cells. ${ }^{7}$ We found that propranolol treatment led to a 7.6-fold reduction in HIF2 $\alpha$ expression in 786-O cells treated with $200 \mu \mathrm{M}$ of propranolol (p < 0.001, Fig. 2E).

\section{Propranolol Slows RCC Tumor Growth in a Xenograft Model and Decreases Serum VEGF-A}

Next, we sought to confirm these results in an in vivo model of $V H L$-deficient tumors. As no established animal model exists for CNS HBs, we utilized an RCC-derived flank xenograft model in athymic nude mice ${ }^{31}$ Mice were 
A
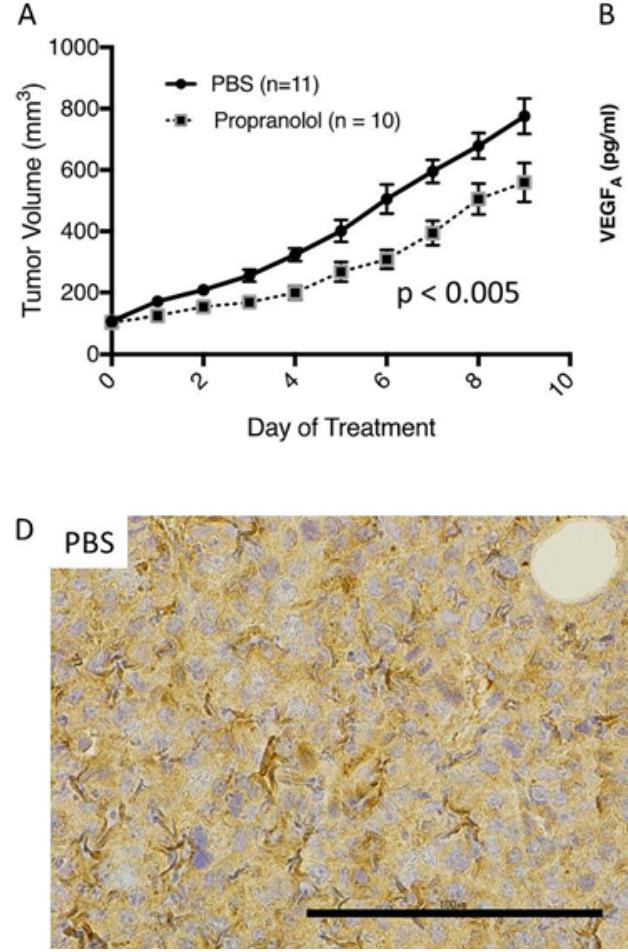
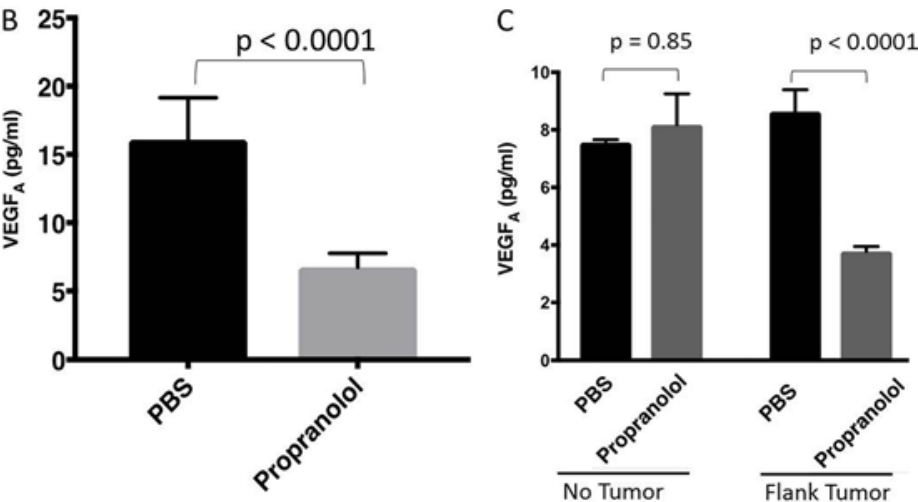

FIG. 3. A: Athymic nude mice bearing 786-O flank tumors have slower rates of tumor progression when treated with propranolol ( $\mathrm{n}$ $=10)$ versus PBS $(n=11, p<0.005)$. B: Circulating VEGF-A levels in propranolol-treated mice are reduced $(p<0.0001)$. Human VEGF-A levels are undetectable in both PBS/propranolol treatment groups. C: An additional cohort of mice $(n=12)$ was treated with PBS or propranolol with $(n=6)$ or without flank tumors $(n=6)$. Circulating murine levels of VEGF-A were not affected by the addition of the tumor $(p=0.85)$. Propranolol treatment decreased the serum levels of murine VEGF-A only in the setting of xenografted 786-O tumors ( $p$ < 0001). D: There was a qualitative decrease in HIF2 $\alpha$ expression in flank tumors, as determined by immunohistochemical analysis in the propranolol-treated mice. Representative photomicrographs are shown (PBS and propranolol, ×40).

randomized to receive daily intraperitoneal injections of propranolol (20 mg/ $\mathrm{kg} / \mathrm{day})$ versus PBS after tumors were between 80 and $150 \mathrm{~mm}^{3}$ in volume. Daily administration of propranolol led to slower tumor xenograft tumor progression, which was maintained through 9 days of propranolol therapy ( $p<0.005)$ (Fig. 3A). Prior to euthanizing mice in the study, peripheral blood and serum were harvested from each mouse and analyzed for VEGF-A levels. Treated mice had a 3-fold reduction in circulating mouse VEGF-A levels as determined by ELISA (Fig. 3B, $\mathrm{p}<0.0001)$. Circulating human VEGF-A levels, which may have potentially been secreted by the tumor, were not detectable in either control or propranolol-treated mice. To confirm these findings, we measured the serum VEGF levels of tumor-bearing mice (treated with PBS) versus mice without xenografted tumors (also treated with PBS). We found no statistically significant difference in the serum VEGF levels of tumor-bearing versus non-tumor-bearing mice (Fig. 3C). Interestingly, non-tumor-bearing mice treated with propranolol had no change in their serum VEGF levels (Fig. 3C). Thus, it appears that propranolol selectively decreases the amount of serum VEGF in the setting of xenografted 786-O tumors.

We further analyzed the expression of GLUT1, VEGF, and HIF $2 \alpha$ in the flank tumors from xenografted mice receiving with PBS versus propranolol. On immunohistochemical analysis, there was no difference in VEGF or GLUT1 expression between both groups. There was a qualitative decrease in the staining for HIF $2 \alpha$, however, which would be consistent with our in vitro western blots (Fig. 3D).

\section{Retrospective Analysis Suggests Propranolol Slows HB Growth in Patients With VHL Disease}

Lastly, we explored whether or not propranolol has any utility in the treatment of patients with VHL disease. We identified 9 patients who were enrolled in an ongoing natural history study of VHL-related HBs at the National Institutes of Health (NCT00005902) who were administered propranolol (for other clinical reasons) for at least 30 days. Dosing ranged between 10 and $100 \mathrm{mg}$ twice daily of propranolol. Of the 9 patients identified, 3 patients had radiographic follow-up data before and after propranolol administration. In all 3 patients, propranolol was started during the longitudinal study for other clinical indications (hypertension, migraine prophylaxis). These 3 patients harbored 66 HBs throughout the CNS. Of the identified tumors, 25 tumors had evidence of growth (at least a 10 $\mathrm{mm}^{3}$ volume increase) prior to implementation of propranolol and were included in subsequent analysis. Tumors in this cohort had a 2.1-year median follow-up on propranolol. The tumors grew slower when patients were taking propranolol (median growth rate $13.3 \mathrm{~mm}^{3} / \mathrm{yr}$ ) than when 
they were not taking propranolol (median growth rate 27.1 $\left.\mathrm{mm}^{3} / \mathrm{yr}, \mathrm{p}<0.0004\right)$. Thus, retrospective clinical data are consistent with our other preclinical data that propranolol may slow HB progression in patients with VHL disease.

\section{Discussion}

VHL disease remains a chronic disorder characterized by the formation of CNS HBs, RCCs, pheochromocytomas, endolymphatic sac tumors, and pancreatic neuroendocrine tumors. ${ }^{5,16,17}$ Despite advances in the understanding of the pathophysiology of tumor formation in patients with VHL, no effective systemic therapies have been developed. Herein, we provide in vitro, in vivo, and retrospective clinical data that suggest that propranolol may be effective in the treatment of VHL-related tumors, including HBs and RCCs.

Propranolol's activity against IHs was first demonstrated clinically. ${ }^{12}$ Following this fortuitous observation, several authors have investigated the mechanism underpinning propranolol's antitumor properties. Propranolol, unlike other beta-receptor antagonists, functions as an inverse agonist against the beta-receptor, a G-protein coupled receptor. ${ }^{4}$ Thus, when bound to the $\beta 1$ or $\beta 2$ receptor, propranolol not only prevents the binding of epinephrine, but also induces a conformational change that downregulates the receptors endogenous activity, thereby decreasing intracellular cAMP (cyclic adenosine monophosphate) levels. ${ }^{4}$ This property of propranolol activity has been suggested by some to underlie propranolol's antiproliferative effects in IHs. Hemangioma stem cells, for example, when exposed to propranolol have been shown to undergo apoptosis, which has been correlated with decreased intracellular cAMP levels with subsequent activation of the extracellular signal-regulated kinase (ERK)1/2 pathway. ${ }^{20}$

Other authors have suggested that propranolol downregulates the PI3-K (phosphatidylinositol 3-kinase) pathway, leading to reduced VEGF levels in $\mathrm{IHs} .^{23}$ In other studies, propranolol exposure decreased IH HIF expression, thereby leading to reduced VEGF expression. ${ }^{7}$ Furthermore, local effects of beta-receptor inhibition leading to vasoconstriction and decreased IH perfusion have been hypothesized as an alternative mechanism of propranololmediated IH regression..$^{20}$ Thus, while the precise mechanism of propranolol activity in IHs remains a subject of ongoing investigation, it appears that propranolol has the potential to 1) induce apoptosis and 2) augment $\mathrm{HIF} /$ VEGF expression while 3) exerting systemic cardiovascular effects that lead to reduced tumor perfusion. . $^{71,20,23}$

Albiñana et al. demonstrated that propranolol reduces primarily cultured HB cells in vitro by inducing apoptosis and augmenting VEGF expression. ${ }^{2}$ Our work confirms these findings in a separate cohort of VHL-derived HB cells and provides further preclinical data that propranolol may be efficacious in treating VHL-HBs and VHL-RCCs. As reported herein, propranolol decreased the viability of cultured HBs while also inducing apoptosis and reducing the viability of an RCC cell line in vitro. Our data further suggest that propranolol is able to decrease HIF2 $\alpha$ expression in 786-O cells. HIF inhibitors have been reported to reverse the VHL phenotype in a zebrafish model of VHL. ${ }^{19}$
In our work, propranolol did not lead to a decrease in 786$\mathrm{O}$ VEGF protein expression, however. One possibility is that VEGF expression would be expected to occur after HIF protein expression was reduced. In IHs, propranolol led to decreased VEGF expression only after 4 days of propranolol exposure. The 786-O cells had limited viability after 48 hours of treatment and thus we may be limited in our ability to detect a significant change in expression of VEGF in 786-O cells treated with propranolol. Furthermore, although one may expect the VEGF mRNA levels to decrease, and not increase, if HIF expression is reduced, this paradoxical finding has been reported in IHs treated with propranolol. ${ }^{7}$

Our work further suggests that propranolol slows RCC tumor progression in vivo while decreasing serum VEGFA levels in treated tumor-bearing mice. In our experiments, human VEGF, which would have otherwise been secreted by the RCC xenograft tumors, was not detected in mouse sera. Additionally, the presence of a 786-O flank tumor did not increase in the serum level of VEGF independently (Fig. 3C). Interestingly, propranolol did not affect the serum VEGF levels of mice that were not bearing 786-O tumors (Fig. 3C). Thus, it appears that propranolol reduced murine VEGF secretion only in the presence of 786-O xenografted tumors. We hypothesize that this may be one reason that propranolol-treated RCC-bearing mice had slower rates of tumor progression. In this way, while propranolol may harbor a direct antitumor effect, it may also induce systemic changes that lead to an inhospitable tumor environment. Our data do not suggest a clear mechanism for why propranolol may reduce serum VEGF selectively in xenografted mice, and further work on this needs to be performed.

Plasma VEGF levels have recently been reported to serve as a biomarker in patients with VHL disease taking propranolol for ocular VHL. ${ }^{1}$ In a recent study, patients with VHL disease who harbored retinal hemangioblastomas received propranolol up to $120 \mathrm{mg} /$ day. Although no retinal HBs regressed in the study, systemic VEGF levels decreased with prolonged treatment with propranolol, which is consistent with our in vivo findings. In our study, the approximate $\mathrm{IC}_{50}$ of propranolol against VHL-HBs and 786-O cells ranged between $50 \mu \mathrm{M}$ and $200 \mu \mathrm{M}$. This is similar to the concentrations at which IHs and VHL-HBs have responded to propranolol therapy in vitro. ${ }^{2,7,38}$ Based on prior pharmacological studies, plasma serum propranolol concentrations would be unlikely to achieve concentrations this high, however. ${ }^{32}$ Nevertheless, although IH growth is inhibited by propranolol treatment between 50 and $300 \mu \mathrm{M}$ in vitro, propranolol has shown clear clinical efficacy for patients with IHs treated with oral propranolol. $7,12,13,38$ Furthermore, a recent study examined the role of VEGF-A expression in 786-O cells. The 786-O cells transfected with siRNA directed against VEGF mRNA had decreased viability, increased rates of apoptosis, and decreased migratory potential. ${ }^{37}$ Similarly, VEGF has been shown to promote the growth of other solid tumors such as colorectal cancer and cervical cancer. ${ }^{27,35}$ Thus, we hypothesize that the reduction in systemic VEGF levels induced by propranolol may be one mechanism underlying propranolol's efficacy in vivo. 
In addition to our in vitro and animal work, our analysis of VHL-HB growth kinetics in a retrospective analysis of a VHL-HB natural history study suggests that patients had slower-growing CNS HBs while receiving propranolol. Although retrospective and derived from a small population of patients, these data are consistent with our cell culture and murine studies and thus provide further biological plausibility that propranolol may have a role in the treatment of VHL-related neoplasms. The protective effect of propranolol and other beta blockers has been reported in other retrospective population-based studies examining hepatocellular carcinoma, prostate cancer, and other malignancies. ${ }^{6,21,26}$ Nevertheless, as HBs have stochastic growth patterns and variable growth rates that can fluctuate over time, further prospective studies are needed to validate propranolol as a viable treatment option for VHL-HBs.

\section{Conclusions}

Overall, our study provides consistent in vitro, in vivo, and retrospective clinical data that propranolol harbors antitumor properties in VHL-HBs and VHL-RCCs, 2 tumors that lead to a majority of morbidity and mortality in patients with VHL disease. ${ }^{5,8,17,34}$ While further work is necessary to identify the mechanisms underlying propranolol's antitumor properties, given the fact that propranolol is a safe, well-tolerated, FDA-approved oral medication that freely crosses the blood-brain barrier, our results encourage the development of clinical trials to test propranolol as a novel treatment agent for VHL disease.

\section{Acknowledgments}

This study was supported by the Intramural Research Program of the National Institute for Neurological Diseases and Stroke, and by the National Institutes of Health Clinical Center in Bethesda, MD. This research was also made possible through the National Institutes of Health Medical Research Scholars Program.

\section{References}

1. Albiñana V, Jiménez Escribano RM, Soler I, RodríguezPadial L, Recio-Poveda L, Villar Gómez de las Heras K, et al: Repurposing propranolol as a drug for the treatment of retinal hemangioblastomas in von Hippel-Lindau disease. Orhanet J Rare Dis 12:122, 2017

2. Albiñana V, Villar Gómez de las Heras K, Serrano-Heras G, Segura T, Perona-Moratalla AB, Mota-Pérez M, et al: Propranolol reduces viability and induces apoptosis in hemangioblastoma cells from von Hippel-Lindau patients. Orphanet J Rare Dis 10:118, 2015

3. Bader HL, Hsu T: Systemic VHL gene functions and the VHL disease. FEBS Lett 586:1562-1569, 2012

4. Baker JG, Hall IP, Hill SJ: Agonist and inverse agonist actions of $\beta$-blockers at the human $\beta_{2}$-adrenoceptor provide evidence for agonist-directed signaling. Mol Pharmacol 64:1357-1369, 2003

5. Butman JA, Linehan WM, Lonser RR: Neurologic manifestations of von Hippel-Lindau disease. JAMA 300:1334-1342, 2008

6. Chang PY, Huang WY, Lin CL, Huang TC, Wu YY, Chen $\mathrm{JH}$, et al: Propranolol reduces cancer risk: a population-based cohort study. Medicine (Baltimore) 94:e1097, 2015

7. Chim H, Armijo BS, Miller E, Gliniak C, Serret MA, Gosain AK: Propranolol induces regression of hemangioma cells through HIF-1 $\alpha$-mediated inhibition of VEGF-A. Ann Surg 256:146-156, 2012
8. Chittiboina P, Lonser RR: Von Hippel-Lindau disease. Handb Clin Neurol 132:139-156, 2015

9. Gossage L, Eisen T, Maher ER: VHL, the story of a tumour suppressor gene. Nat Rev Cancer 15:55-64, 2015

10. Joshi S, Singh AR, Durden DL: Pan-PI-3 kinase inhibitor SF1126 shows antitumor and antiangiogenic activity in renal cell carcinoma. Cancer Chemother Pharmacol 75:595608, 2015

11. Latif F, Tory K, Gnarra J, Yao M, Duh FM, Orcutt ML, et al: Identification of the von Hippel-Lindau disease tumor suppressor gene. Science 260:1317-1320, 1993

12. Léauté-Labrèze C, Dumas de la Roque E, Hubiche T, Boralevi F, Thambo JB, Taïeb A: Propranolol for severe hemangiomas of infancy. N Engl J Med 358:2649-2651, 2008

13. Léauté-Labrèze C, Hoeger $P$, Mazereeuw-Hautier J, Guibaud L, Baselga E, Posiunas G, et al: A randomized, controlled trial of oral propranolol in infantile hemangioma. N Engl J Med 372:735-746, 2015

14. Li P, Guo Z, Gao Y, Pan W: Propranolol represses infantile hemangioma cell growth through the $\beta 2$-adrenergic receptor in a HIF-1 $\alpha$-dependent manner. Oncol Rep 33:3099-3107, 2015

15. Littell RC: Repeated measures analysis with clustered subjects, in Proceedings of the SAS Global Forum 2007 Conference. Cary, NC: SAS Institute, 2007 (http://www2.sas. com/proceedings/forum2007/178-2007.pdf) [Accessed June 27, 2018]

16. Lonser RR, Butman JA, Huntoon K, Asthagiri AR, Wu T, Bakhtian KD, et al: Prospective natural history study of central nervous system hemangioblastomas in von HippelLindau disease. J Neurosurg 120:1055-1062, 2014

17. Lonser RR, Glenn GM, Walther M, Chew EY, Libutti SK, Linehan WM, et al: von Hippel-Lindau disease. Lancet 361:2059-2067, 2003

18. Lundin P, Pedersen F: Volume of pituitary macroadenomas: assessment by MRI. J Comput Assist Tomogr 16:519-528, 1992

19. Metelo AM, Noonan HR, Li X, Jin Y, Baker R, Kamentsky L, et al: Pharmacological HIF2 $\alpha$ inhibition improves VHL disease-associated phenotypes in zebrafish model. J Clin Invest 125:1987-1997, 2015

20. Munabi NCO, England RW, Edwards AK, Kitajewski AA, Tan QK, Weinstein A, et al: Propranolol targets hemangioma stem cells via cAMP and mitogen-activated protein kinase regulation. Stem Cells Transl Med 5:45-55, 2016

21. Nkontchou G, Aout M, Mahmoudi A, Roulot D, Bourcier V, Grando-Lemaire V, et al: Effect of long-term propranolol treatment on hepatocellular carcinoma incidence in patients with HCV-associated cirrhosis. Cancer Prev Res (Phila) 5:1007-1014, 2012

22. Omar AI: Bevacizumab for the treatment of surgically unresectable cervical cord hemangioblastoma: a case report. J Med Case Reports 6:238, 2012

23. Pan WK, Li P, Guo ZT, Huang Q, Gao Y: Propranolol induces regression of hemangioma cells via the down-regulation of the PI3K/Akt/eNOS/VEGF pathway. Pediatr Blood Cancer 62:1414-1420, 2015

24. Park DM, Zhuang Z, Chen L, Szerlip N, Maric I, Li J, et al: von Hippel-Lindau disease-associated hemangioblastomas are derived from embryologic multipotent cells. PLoS Med 4:e60, 2007

25. Peng S, Shepard MJ, Wang J, Li T, Ning X, Cai L, et al: Genotype-phenotype correlations in Chinese von Hippel-Lindau disease patients. Oncotarget 8:38456-38465, 2017

26. Perron L, Bairati I, Harel F, Meyer F: Antihypertensive drug use and the risk of prostate cancer (Canada). Cancer Causes Control 15:535-541, 2004

27. Qi L, Xing LN, Wei X, Song SG: Effects of VEGF suppression by small hairpin RNA interference combined with ra- 
diotherapy on the growth of cervical cancer. Genet Mol Res 13:5094-5106, 2014

28. Rogers LR, LoRusso P, Nadler P, Malik G, Shields A, Kaelin W: Erlotinib therapy for central nervous system hemangioblastomatosis associated with von Hippel-Lindau disease: a case report. J Neurooncol 101:307-310, 2011

29. Sardi I, Sanzo M, Giordano F, Buccoliero AM, Mussa F, Aricò $\mathrm{M}$, et al: Monotherapy with thalidomide for treatment of spinal cord hemangioblastomas in a patient with von HippelLindau disease. Pediatr Blood Cancer 53:464-467, 2009

30. Sizdahkhani S, Feldman MJ, Piazza MG, Ksendzovsky A, Edwards NA, Ray-Chaudhury A, et al: Somatostatin receptor expression on von Hippel-Lindau-associated hemangioblastomas offers novel therapeutic target. Sci Rep 7:40822, 2017

31. Stiles JM, Amaya C, Rains S, Diaz D, Pham R, Battiste J, et al: Targeting of beta adrenergic receptors results in therapeutic efficacy against models of hemangioendothelioma and angiosarcoma. PLoS One 8:e60021, 2013

32. Von Bahr C, Hermansson J, Tawara K: Plasma levels of (+) and (-)-propranolol and 4-hydroxypropranolol after administration of racemic ( \pm )-propranolol in man. Br J Clin Pharmacol 14:79-82, 1982

33. Wanebo JE, Lonser RR, Glenn GM, Oldfield EH: The natural history of hemangioblastomas of the central nervous system in patients with von Hippel-Lindau disease. J Neurosurg 98:82-94, 2003

34. Wilding A, Ingham SL, Lalloo F, Clancy T, Huson SM, Moran A, et al: Life expectancy in hereditary cancer predisposing diseases: an observational study. J Med Genet 49:264-269, 2012

35. Xing X, Gu X, Ma T, Ye H: Biglycan up-regulated vascular endothelial growth factor (VEGF) expression and promoted angiogenesis in colon cancer. Tumour Biol 36:1773-1780, 2015

36. Yang C, Huntoon K, Ksendzovsky A, Zhuang Z, Lonser RR: Proteostasis modulators prolong missense VHL protein activity and halt tumor progression. Cell Reports 3:52-59, 2013

37. Zeng FC, Zeng MQ, Huang L, Li YL, Gao BM, Chen JJ, et al: Downregulation of VEGFA inhibits proliferation, promotes apoptosis, and suppresses migration and invasion of renal clear cell carcinoma. OncoTargets Ther 9:2131-2141, 2016

38. Zhang L, Mai HM, Zheng J, Zheng JW, Wang YA, Qin ZP, et al: Propranolol inhibits angiogenesis via down-regulating the expression of vascular endothelial growth factor in hemangioma derived stem cell. Int J Clin Exp Pathol 7:48-55, 2013

\section{Disclosures}

The authors report no conflict of interest concerning the materials or methods used in this study or the findings specified in this paper.

\section{Author Contributions}

Conception and design: Chittiboina, Shepard, Zhuang. Acquisition of data: Chittiboina, Shepard, Bugarini, Edwards, Lu, Zhang. Analysis and interpretation of data: all authors. Drafting the article: Chittiboina, Shepard, Bugarini, Edwards, Wu, Zhuang. Critically revising the article: Chittiboina, Shepard, Bugarini, $\mathrm{Wu}$, Zhuang. Reviewed submitted version of manuscript: all authors. Approved the final version of the manuscript on behalf of all authors: Chittiboina. Statistical analysis: Shepard, Bugarini, Lu, Wu. Administrative/technical/material support: Chittiboina, Zhuang. Study supervision: Chittiboina, Zhuang.

\section{Supplemental Information \\ Previous Presentations}

Portions of this manuscript were previously presented as posters at the 2017 AANS Annual Scientific Meeting, Los Angeles, California, April 22-26, 2017, and at the 22nd Annual Meeting and Education Day, Society of Neuro-Oncology, San Francisco, California, November 16-19, 2017.

\section{Correspondence}

Prashant Chittiboina: National Institute of Neurological Diseases and Stroke, National Institutes of Health, Bethesda, MD. prashant. chittiboina@nih.gov. 